\title{
ASPECTOS BIOÉTICOS Y JURÍDICOS DEL ACOMPAÑAMIENTO EN LA LEY CHILENA DE INTERRUPCIÓN VOLUNTARIA DEL EMBARAZO EN TRES CAUSALES
}

\begin{abstract}
Verónica Undurraga Valdés ${ }^{2}$
Resumen: La ley que regula la despenalización de la interrupción voluntaria del embarazo en tres causales contempla un programa de acompañamiento que garantiza el derecho a la autonomía de la paciente. En la práctica, sin embargo, no todos los modelos de acompañamiento que se ofrecen en Chile cumplen con este estándar normativo. El artículo analiza el acompañamiento desde las perspectivas jurídica y bioética, y explora las características que debiera tener un programa de acompañamiento respetuoso de la autonomía de la mujer embarazada. El texto concluye afirmando que el profesional de salud que ofrece acompañamiento debe involucrarse en un diálogo respetuoso y genuino con la paciente, proveer información adecuada, abstenerse de imponer su propia interpretación sobre la experiencia de la mujer, apoyarla en su deliberación y asumir que las preferencias y valores de la mujer deben guiar el proceso de toma de decisiones. Los programas que no cumplen con estos estándares pueden estar infringiendo obligaciones legales.
\end{abstract}

Palabras clave: acompañamiento, aborto, interrupción del embarazo, autonomía del paciente

\section{Bioethical and legal aspects of abortion counseling under Chilean law}

\begin{abstract}
The Chilean statute decriminalizing abortion under three specific grounds provides for a counseling program which guarantees patient's autonomy. However, the reality is that not all counseling programs that are offered in Chile comply with this normative standard. The article analyzes abortion counseling from a legal and bioethical perspective, and examines the features of a counseling program respectful of the pregnant woman's autonomy. The text concludes stating that the health care professional that offers support he/she should be involved in a respectful and genuine dialogue with the patient, provide adequate information, refraining from imposing his/her own interpretation about the experience of the woman, support her in deliberating and assume that her preferences and values must guide the process of decision making. The programs which do not fulfill these standards may be contravening legal obligations.
\end{abstract}

Key words: counseling, abortion, legal interruption of pregnancy, patient's autonomy

Aspectos bioéticos e jurídicos do acompanhamento da lei chilena de interrupçáo voluntária da gravidez em três causas

Resumo: A lei que regula a descriminalização da interrupção voluntária de gravidez em três causal inclui um programa de acompanhamento que garante o direito à autonomia do paciente. Na prática, no entanto, nem todos os modelos de acompanhamentos oferecidos no Chile cumprem com esse padrão normativo. $\mathrm{O}$ artigo analisa o acompanhamento com base nas perspectivas jurídicas e bioética e explora as características que devem ter um programa de apoio respeitoso da autonomia da mulher grávida. $\mathrm{O}$ texto conclui afirmando que o profissional de saúde que fornece acompanhamento deve estabelecer um diálogo genuíno e respeitoso com a paciente, fornecer informaçôes adequadas, abster-se de impor sua própria interpretação sobre a experiência da mulher, apoiá-la em sua deliberação e assumir que as preferências e os valores da mulher devem orientar o processo de tomada de decisão. Os programas que náo cumpram com estas normas podem estar infringindo obrigaçóes legais.

Palabras clave: acompañamiento, aborto, interrupción del embarazo, autonomía del paciente

\footnotetext{
${ }^{1}$ Este artículo forma parte del proyecto de investigación titulado "El libre desarrollo de la personalidad como derecho fundamental en el Derecho chileno, comparado e internacional de los derechos humanos". CONICYT, Fondecyt de Iniciación en Investigación 2014, Proyecto No 11140853.

${ }^{2}$ Facultad de Derecho, Universidad Adolfo Ibáńez, Chile

Correspondencia: veronica.undurraga@uai.cl
} 


\section{Introducción}

Una de las particularidades de la discusión legislativa chilena sobre despenalización de la interrupción voluntaria del embarazo (Ley IVE)(1), si se la compara con similares procesos en otros países, fue la relevancia que adquirió el tema del acompańamiento para las mujeres que se encuentren bajo alguna de las tres causales de interrupción del embarazo que contempla la ley (2, sesión 82, legislatura 364:743) ${ }^{3}$. Aunque el acompañamiento quedó explícitamente asociado al reconocimiento de la autonomía de la paciente para decidir si continuar o interrumpir su embarazo, solo algunos de los programas de acompañamiento que existen en Chile son compatibles con esta exigencia legal. Durante el proceso legislativo el acompańamiento se presentó inicialmente como la contrapropuesta a la despenalización. Se afirmaba que había que mantener el tipo penal, pero ofrecerle acompañamiento a la mujer. Con excepción del modelo presentado por el gobierno, los programas de acompañamiento que se expusieron en el Congreso tienen como propósito explícito disuadir a la mujer de abortar y sus creadores se opusieron a la despenalización $(2)^{4}$. Aunque existen modelos de acompañamiento diseñados para países con regímenes de aborto legal, esos programas no fueron analizados durante el proceso legislativo $(3,4,5)^{5}$. Este artículo desarrolla las características que debiera tener un programa de acompañamiento compatible con una ley que califica la interrupción del embarazo bajo las tres causales como una

\footnotetext{
${ }^{3}$ La aprobación del proyecto de ley solo pudo destrabarse con el voto de la senadora Goic, el que fue condicionado a que el gobierno comprometiera un fortalecimiento del programa de acompańamiento.

${ }^{4}$ En la Historia de la Ley 21.030(1) pueden consultarse las exposiciones de los programas de Fundación Chile Unido, Acompañar-es, Corporación Maternitas y la propuesta de acompañamiento presentada por el abogado Gonzalo García.

${ }^{5}$ A modo de ejemplo, se citan algunos de estos modelos en las referencias(3-5). En la discusión legislativa se hicieron algunas menciones al modelo alemán para argumentar a favor de una consejería obligatoria y disuasiva del aborto. Sin embargo, no se aclaró que en Alemania el modelo legal de acompańamiento es obligatorio solo cuando se solicita el aborto sin mención de causal dentro de las primeras 12 semanas de embarazo y que es voluntario en el caso de concurrencia de causales médicas o criminológicas. Aunque la ley plantea como objetivo de la consejería proteger la vida prenatal, es explícita en señalar que "el propósito de la consejería no es presionar a la mujer a hablar o a cooperar", que la consejería "debe conducirse de manera de dejar la decisión abierta y bajo la premisa de que la responsabilidad de la decisión recae en la mujer embarazada. La consejería debe ser alentadora y comprensiva y no reprobatoria y condescendiente". Ver referencia (5).
}

legítima prestación de salud, y en que el derecho de las mujeres a optar por la interrupción está protegido, como lo señaló el Tribunal Constitucional, bajo los derechos que la Constitución reconoce a la mujer a la libertad y a la mayor realización material y espiritual posible, a la igualdad, a la integridad física y síquica, a la privacidad y a la salud $(6$, considerando 35).

\section{El derecho a la autonomía de la persona en su atención de salud}

La Ley IVE es neutra, en el sentido que no favorece ni la decisión de interrumpir ni la de mantener un embarazo, pero no es neutra respecto de su calificación del acto de interrupción del embarazo, ni tampoco de las características del acompañamiento. En el Derecho chileno, la interrupción del embarazo bajo las tres causales legales es una legítima prestación de salud y un acto lícito, esto es, conforme a derecho. La Ley IVE exige que el programa de acompańamiento sea voluntario, respetuoso de la autonomía de la mujer, personalizado, basado en información veraz y objetiva y no inductivo ni a la decisión de mantener el embarazo ni a la de interrumpirlo(1).

El reconocimiento de la autonomía personal forma parte de los presupuestos básicos del Derecho, de la forma en que entendemos que este funciona y de la idea de "persona" que lo sustenta. La sola idea de que las personas tienen derechos supone que son agentes que pueden relacionarse con otros desde una posición jurídica, que asegura el respeto mutuo, y no como simples beneficiarias de la buena voluntad de otros(7:51-53).

El Tribunal Constitucional, los tribunales de justicia y la doctrina han empezado a usar en Chile la expresión "derecho al libre desarrollo de la personalidad", utilizada en algunos países europeos y de América Latina para referirse al derecho a la autonomía personal(8). De acuerdo al Tribunal Constitucional, el libre desarrollo de la personalidad constituye "una expresión de la dignidad de toda persona, que se encuentra afirmada enfáticamente en el inciso primero del artículo $1^{\circ}$ de la Carta Fundamental" (9, considerando 51). Dependiendo de la argumentación que adopten los distintos tribunales y autores, el derecho de autonomía o libre desarrollo de la personalidad se ha 
adscrito al artículo $1^{\circ}$ antes citado, al artículo 19 $\mathrm{n}^{\circ} 4$, que reconoce el derecho a la vida privada; al artículo $19 \mathrm{n}^{\circ} 7$, que establece el derecho a la libertad personal y seguridad individual, y también se ha inferido del conjunto de normas constitucionales que reconocen libertades específicas(8). El artículo $1^{\circ}$ inciso 4 de la Constitución se refiere al deber general del Estado de "contribuir a crear las condiciones sociales que permitan a cada uno de los integrantes de la comunidad nacional su mayor realización espiritual y material posible." Una de las formas de cumplir con este deber es dictando leyes y regulaciones administrativas que hagan operativo el derecho a la autonomía en ámbitos específicos. La Ley 20.584, que regula los derechos y deberes que tienen las personas en relación con acciones vinculadas a su atención en $\operatorname{salud}(10)$, es la norma que en Chile incorpora explícitamente el derecho a la autonomía personal en la atención de salud. Por su parte, la Ley IVE contiene una serie de reglas que aseguran el derecho a la autonomía de la mujer que se encuentra en alguna de las tres causales legales de interrupción del embarazo, para decidir si continuar o interrumpir la gestación(1).

Aunque existen muchos otros ámbitos en los que se regulan manifestaciones específicas del derecho a la autonomía personal, el de la atención de salud es particularmente importante. El cuerpo es el referente primero y más básico de la propia identidad. Por eso, existe un vínculo estrecho entre el derecho a la autonomía y el derecho a la seguridad personal, reconocida en el artículo 19 No7 de la Constitución Política. Las personas sienten amenazada su autonomía al percibir que pierden el control sobre lo que se les hace a sus cuerpos. Esta percepción se intensifica si las intervenciones médicas afectan aspectos íntimos y muy centrales en las definiciones de vida de las personas, como el ejercicio de la sexualidad y las decisiones reproductivas. A esto hay que agregar que la enfermedad puede dejar a las personas vulnerables y en una situación de dependencia, situación que puede agravarse cuando existe desbalance de poder entre pacientes y médicos, por las diferencias de conocimientos u otras variables como el género o la situación de pobreza.

Como una forma de reconocer el igual estatus moral de todas las personas y evitar el paternalismo injustificado, el Derecho adscribe autonomía moral a todos los adultos. Salvo prueba de incapacidad o especial coerción, en la atención de salud se considera que toda persona adulta es capaz de discernir lo que es correcto para ella y actuar conforme a esa convicción. Los menores de edad son sujetos de derecho que van ejerciendo su autonomía en forma progresiva. La autoridad parental existe en exclusivo beneficio de las niñas, niños y adolescentes y va limitándose gradualmente hasta que las condiciones de madurez habilitan a los adolescentes a actuar por sí mismos, excluyendo la representación parental $(11,12)$.

En la Ley IVE, el reconocimiento del derecho a la autonomía de la paciente se manifiesta en las reglas que exigen la entrega de información veraz, completa, objetiva y pertinente a la mujer para que ella pueda tomar una decisión informada; en el hecho que sea la mujer la que tiene la última palabra sobre si continuar o interrumpir un embarazo que califique bajo las causales; en la exigencia de acreditación de la voluntad expresa, previa y por escrito de la mujer o niña para que los médicos puedan proceder a la interrupción de su embarazo, incluso en casos de riesgo vital; en la obligación de escuchar a las niñas y adolescentes, en los resguardos que la ley contempla frente a situaciones de coacción, en las garantías legales establecidas para evitar que la objeción de conciencia de los profesionales de salud constituya un obstáculo al ejercicio de la decisión de la mujer y, por último, en las características que la ley estableció para el programa de acompañamiento(1).

La Ley IVE ordena que el acompañamiento solo se realice en la medida en que la mujer lo autorice y que sea respetuoso de su libre decisión(1). El Tribunal Constitucional rechazó el argumento de que el acompañamiento debía ser obligatorio y disuasivo de la decisión de interrumpir el embarazo(6, considerandos 6,111 y 120). La mujer puede poner término al programa de acompañamiento que haya aceptado recibir, como también solicitar acceso al programa aunque inicialmente lo hubiere rechazado(13), y puede elegir libremente tanto la entidad como el programa de acompańamiento que estime más adecuado a su situación particular y convicciones personales $(1,13)$. El acompańamiento busca ofrecer las mejores condiciones posibles para el ejercicio de la autonomía de la mujer: provisión de la información relevante, escucha 
atenta y apoyo no directivo en el discernimiento, contención emocional en un espacio seguro y libre de coerción, y ayuda económica y social que pueda dar viabilidad y hacer menos gravosa la decisión que la mujer adopte(13).

Desde una mirada cultural, Chile aún está transitando desde un modelo de paternalismo médico a uno fundado en el respeto a la autonomía del paciente(14). Bajo el modelo de paternalismo médico que primó hasta avanzado el siglo veinte, las decisiones sobre los tratamientos y cuidados de salud de las personas eran adoptadas por los médicos, basados en sus conocimientos y guiados por el deber de actuar en beneficio del paciente. La relación entre médico y paciente se fundaba en el compromiso responsable del médico y en la confianza por parte del paciente sobre el buen proceder profesional y criterio de aquel.

En cambio, bajo el modelo fundado en el respeto a la autonomía, el principio de beneficencia debe siempre vincularse con el principio de autonomía, cuando los pacientes están en condiciones de expresar sus preferencias y valores. Si no pueden hacerlo, estas preferencias y valores pueden indagarse en un diálogo con las personas que más conocen al paciente. La autonomía personal se define como autogobierno. El derecho a la autonomía protege el interés que tiene una persona de vivir de acuerdo a las creencias, razones y valores que ella reconoce como auténticamente propios, y no bajo influencias o factores que socavan o destruyen su habilidad de actuar como una persona única $(15,16)$. El respeto a la autonomía del paciente requiere que el personal médico no solo muestre una actitud de respeto, sino que efectivamente actúe respetuosamente permitiendo a la persona tomar decisiones sobre su propia salud(17:106-107). La vulneración de esta obligación se genera por actitudes y acciones que ignoran, ofenden, humillan o no atienden al derecho de los pacientes de actuar autónomamente(17:107). El respeto a la autonomía del paciente no solo implica una obligación de abstenerse de impedir el ejercicio de autonomía del paciente, sino que, en ocasiones, que el personal de salud deba adoptar medidas que permitan o faciliten a la persona ese ejercicio.

No es siempre fácil para los médicos adherir al principio de autonomía de los pacientes, especial- mente cuando consideran que las decisiones de éstos son irracionales o equivocadas bajo los criterios médicos, y que van en contra de lo que el médico percibe como la decisión más beneficiosa para el paciente. Algunos médicos piensan que el énfasis en la autonomía del paciente puede perjudicar al propio paciente, al recargarlo con una responsabilidad excesiva o porque el énfasis en la autonomía erosiona la relación de confianza que debe existir entre el paciente y su médico(18).

El problema de una mirada paternalista es que el cuidado de la salud puede vincularse con aspectos íntimos y centrales de la vida de la persona y es muy difícil para otro individuo tomar decisiones que resulten compatibles con el sistema de valores del paciente y que, por lo tanto, aseguren el respeto a sus intereses y su bienestar(19:21). No hay que olvidar que es el paciente y no el profesional de salud quien debe vivir con las consecuencias de la decisión.

En las sociedades pluralistas contemporáneas es muy difícil encontrar criterios sustantivos compartidos sobre lo que se considera una buena vida o el actuar correcto. Como consecuencia, la bioética se ha volcado a desarrollar procedimientos de toma de decisión que asumen que las personas involucradas tienen diferentes sistemas de valores(20). En pocas décadas, la autonomía personal ha pasado a transformarse en un valor preeminente de la bioética occidental. La autonomía del paciente, correctamente entendida, no erosiona las relaciones de confianza. Por el contrario, posibilita una confianza genuina y una profundización del vínculo, al situar al paciente en un plano de igualdad y no en el rol de mero beneficiario. Respetar la autonomía del paciente requiere un esfuerzo de diálogo real y constante que ha sido inusual hasta ahora en el ejercicio de la medicina. Lo que se busca con el diálogo es llegar a una decisión compartida entre el profesional de salud y su paciente, que esté libre de imposiciones y de silenciamientos. En los casos en que ese consenso resulta imposible, persiste el objetivo de lograr una comprensión mutua de la postura del otro y el respeto por sus razones y sentimientos(21:186). En ambas circunstancias se preservan las condiciones necesarias para la autonomía. Adicionalmente, lo que asegura el derecho a la autonomía es que, ante la imposibilidad de alcanzar una decisión compartida, en razón de la 
discrepancia de valores, la estructura del proceso de decisiones otorgue al paciente la autoridad última para identificar aquellos que deben guiar la decisión sobre su tratamiento(20). Sin embargo, la existencia de esta regla de competencia no debe ocultar lo más importante: el desafío de aprender una nueva forma de comunicación con el paciente que, aplicando el principio de beneficencia, lo haga respetando su autonomía.

Las resistencias a respetar la autonomía de los pacientes se agudizan cuando concurren factores que hacen desconfiar al personal de salud de la capacidad o aptitud moral de los pacientes para tomar decisiones correctas. Hay evidencia que demuestra que esta desconfianza a veces se sustenta en estereotipos que operan inconscientemente $(22,23: 40)$. Un estereotipo es una preconcepción sobre las características o atributos que posee un grupo de la población y que hace que se asuma que un determinado individuo, porque pertenece o porque se cree que pertenece a ese grupo, necesariamente los tiene. En consecuencia, se presume que la persona, por pertenecer a ese grupo, siente y actúa (o debe actuar) como se entiende que lo hace el grupo al que se le adscribe, sin tomar en consideración las capacidades y características de esa persona específica. Las mujeres resultan particularmente afectadas por la operación de estereotipos de género en contextos de toma de decisiones. Nuestra cultura entiende las características de la masculinidad y la femineidad como binarios opuestos y jerarquizados(24). Las capacidades asociadas a la toma de decisiones, como ser racional, independiente, fuerte, proactivo, objetivo, imparcial y capaz de desarrollar pensamiento abstracto, culturalmente se vinculan a la masculinidad. Sus opuestos, la irracionalidad, dependencia, pasividad, debilidad, subjetividad, parcialidad, emocionalidad y el pensamiento concreto, se consideran atributos femeninos. Los dualismos están jerarquizados porque lo femenino se define como déficit: la irracionalidad es la ausencia de racionalidad, la dependencia es el déficit de independencia, etc., y los atributos adscritos a los hombres son además valorados como superiores en casi todas las actividades humanas que otorgan mayor estatus social(24). Los estereotipos no necesariamente son hostiles hacia las mujeres cuando se trata de labores de cuidado. También son estereotipos cul- turales que las mujeres son acogedoras, maternales, generosas, sacrificadas y el pilar moral de la familia. Sin embargo, también estos estereotipos benévolos, porque funcionan como prescripciones sobre cómo deben ser las mujeres y las encasillan exclusivamente en roles de cuidado, limitan el ejercicio de su autonomía(22:17).

La razón por la cual la operación de estereotipos puede ser dañina en el contexto de la atención de salud es precisamente porque la paciente individual se hace invisible tras el estereotipo a través del cual está siendo evaluada. En lugar de ser tratada como una persona única, con su contexto específico, es atendida asumiendo que sus valores, necesidades, deseos, comportamientos y circunstancias de vida corresponden a las del estereotipo en que fue encasillada(23:334). Si el derecho a la autonomía en el ámbito de la salud garantiza a la persona la posibilidad de tomar decisiones en consideración de sus propios motivos, razones y valores, en el contexto de la decisión de continuar o interrumpir un embarazo la operación de estereotipos de género, incluso aquellos aparentemente benevolentes, puede afectar profundamente la autonomía de la mujer. Lo que define el bienestar de una persona excede los aspectos puramente médicos en los que el personal de salud puede aportar una opinión y depende, en gran medida, de las características de esa persona única.

Los programas de acompañamiento que asumen que siempre la mejor decisión para la mujer es mantener el embarazo, independientemente de las circunstancias, se sustentan en visiones estereotipadas de las mujeres. Sus proponentes afirmaron en la discusión legislativa que ninguna mujer que decide abortar está actuando autónomamente y que, por eso, necesita que la sociedad intervenga para evitar que tome una decisión equivocada y contraria a su propio bienestar. Las razones serían que la mujer no podría razonar bien (está muy emocional), que está siendo presionada por otros para abortar (no piensa por sí misma, su voluntad es voluble, es una persona débil) y que una mujer, si tuviera las condiciones adecuadas para ejercer la maternidad y fuera realmente libre, siempre optaría por ser madre (el valor y destino de la mujer está en la maternidad)(2:191-375,33:333).

El supuesto en que se basan los programas de 
acompañamiento disuasivo, de que el bienestar de las mujeres necesariamente va asociado a la decisión de mantener el embarazo incluso bajo situaciones extremas, debe ser objeto de un escrutinio cuidadoso. Aceptarlo implica afirmar que la decisión de una mujer de interrumpir su embarazo va a ser siempre y necesariamente una decisión equivocada, porque es contraria a sus propios intereses, definidos éstos no por ella, sino por el médico o el equipo de salud. Se afirma que el aborto daña psicológicamente a la mujer. Sin embargo, este argumento ha sido convincentemente rebatido al probarse que los estudios que sirven de fuente a esta afirmación tienen deficiencias metodológicas(25). En todo caso, el Tribunal Constitucional reconoció la existencia de la controversia metodológica, pero determinó que, en cualquier caso, corresponde a la mujer decidir si asume los eventuales riesgos(6, considerando 99).

Es precisamente en la definición de cuáles son los intereses relevantes para la mujer en los que los estereotipos de género operan justificando el paternalismo. Por un lado, se subestima la intensidad e importancia de los daños que puede significar para la mujer la mantención del embarazo, porque está normalizada la idea de la autopostergación y el sacrificio maternal; por otro, solo se visibilizan como beneficios para la mujer aquellos relacionados con la maternidad (aunque sea impuesta), sin tomar en consideración la multiplicidad de proyectos y relaciones que involucra un plan de vida que, eventualmente, puede verse afectado por la decisión de mantener el embarazo, por ejemplo, cuando este ha sido producto de una violación.

Es posible que la mejor decisión para algunas mujeres que se encuentran en las tres causales sea mantener su embarazo. Pero no es posible que sea la mejor decisión para todas las mujeres. Cada mujer, siguiendo la regla general aplicable a todo paciente en relación a su atención de salud, es quien debe ponderar, de acuerdo con sus razones, valores y creencias, la importancia que va a atribuir a los riesgos y beneficios de las intervenciones médicas disponibles para su situación y adoptar una decisión informada y autónoma. El Tribunal Constitucional afirmó que "la mujer no puede ser considerada como un instrumento utilitario de protección del no nacido. El legislador (...) no puede ir contra la mujer imponiendo su voluntad e incluso poniendo en peligro su vida o imponiéndole deberes más allá de lo que a cualquier persona se le exige" (6, considerando 47).

La autonomía debe respetarse independientemente de la opinión que tenga el equipo de salud sobre la interrupción del embarazo. Aunque este artículo critica los programas de acompañamiento cuyo objetivo es disuadir de la decisión de abortar por ser contrarios a la ley, si existieran programas que buscaran inducir a abortar también serían ilegales.

\section{Acompañamiento respetuoso de la autonomía de la paciente}

Un programa de acompañamiento respetuoso de la autonomía de la mujer requiere de una actitud de reconocimiento que permita a ésta presentarse sin vergüenza, confiar en su propia competencia y sentirse responsable de sus decisiones. Debe garantizar privacidad, confidencialidad, acogida y seguridad. Un acompañamiento efectivo debe crear las condiciones para que la mujer se sienta autónoma(26:136). Esto significa que sienta que tiene poder, entendido no como poder sobre otros, sino como la capacidad de perseguir sus planes de vida y de afirmar su propio valor(21:204). Crear estas condiciones requiere preocuparse desde la disposición del espacio físico en que se lleva adelante el acompañamiento, hasta de las formas de comunicación, tanto el lenguaje explícito como corporal de los miembros del equipo de acompañantes. El equipo debe trabajar sus propios sesgos inconscientes para evitar que la operación de estereotipos respecto de la mujer interfiera en su trabajo. El equipo de salud debe estar atento a la eventual coerción que individuos de su círculo puedan ejercer sobre ella.

En segundo lugar, la mujer debe recibir información clara, veraz, objetiva, pertinente, oportuna y de calidad, en un lenguaje que facilite su comprensión. El modo en que se entrega la información es clave. Dada la autoridad que tiene el profesional de salud en nuestra sociedad y la forma tradicional en que se comunican con los pacientes, es común que éstos entiendan información que es descriptiva como imperativa e incuestionable(21:189). El profesional de salud debe ser plenamente consciente de que la autoridad y poder normalmente son invisibles para quien los posee. 
Por lo tanto, debe ser capaz de evaluar la situación comunicativa desde el punto de vista de la paciente. En el acompańamiento, la información y la forma en que se entregue debe permitir a la mujer comprender que tiene alternativas y esclarecerlas. Debe poder entender los pasos a seguir en caso de optar por la continuación o la interrupción del embarazo. La información permite el ejercicio de la autonomía, porque la persona, para poder actuar autónomamente, necesita tener algún grado de control real sobre su propia situación y tener un rango de opciones posibles. En el proceso de acompañamiento se despliegan las alternativas y se apoya las capacidades de la mujer para evaluarlas. Corresponde al equipo de salud acompañar a la mujer en la identificación de los recursos de apoyo con los que cuenta.

En tercer lugar, el acompañamiento debe facilitar que la mujer se conecte con su propia experiencia, emociones, sentimientos y valores. El equipo a cargo del acompañamiento debe tener para esto competencia técnica y habilidades específicas. Para lograr que la mujer pueda conectarse con lo que está viviendo, quien acompaña debe abstenerse de emitir juicios y evitar imponer interpretaciones sobre la experiencia que la mujer está teniendo(3:15). El ejercicio de la autonomía requiere que la persona pueda sacar su propia voz y formar su propio relato sobre lo que vive. Ese relato debe ser validado y no reinterpretado o corregido por el equipo de acompañamiento(3:15,30;4:51). La validación del relato de la mujer es especialmente importante, considerando el estigma que afecta a las mujeres que plantean la posibilidad de interrumpir sus embarazos. El estigma opera impidiendo la aceptación social de la persona estigmatizada, marginándola, desvalorizándola, de manera que queda desacreditada ante un mundo que la rechaza $(27,28)$. La validación del relato de la mujer busca liberarla de la internalización del estigma, es decir, evitar que incorpore las percepciones o creencias negativas de otros en su manera de verse a sí misma, afectando su autoestima y generando sentimientos de culpa o vergüenza(28). La realidad del estigma debe ser profesionalmente enfrentada, especialmente en la evaluación de la concurrencia de la causal de violación por parte del equipo de salud. La validación del relato de la mujer se justifica en la idea de que las personas que son juzgadas por la sociedad en forma negativa van a encontrar muchas dificultades para aceptar sus emociones y respetar sus propias creencias, en sentir que ellas o sus proyectos son valiosos. Para aceptar el valor y el sentido de sus acciones, las personas necesitan un horizonte semántico y simbólico en que esas acciones se consideren siquiera posibles $(28,29)$. En la práctica, la validación en el procedimiento de acompańamiento se logra con una comunicación que transmita a la mujer que su situación, sus emociones y sus pensamientos no son anormales y que son compartidos por otras mujeres. Cuando la mujer percibe que sus sentimientos y pensamientos son aceptados por quien la acompaña, puede indagar en ellos y está en mejores condiciones para tomar una decisión verdaderamente autónoma.

En cuarto lugar, el acompañamiento debe enfocarse en facilitar a la mujer la identificación de los recursos personales, familiares y sociales de apoyo de que dispone, con el fin de que ella pueda planificar una estrategia para afrontar la situación futura, cualquiera sea la decisión que tome. Esta estrategia debe ser específica para cada mujer, atendiendo sus necesidades, circunstancias, la causal de interrupción del embarazo, su edad, entre otros factores. De esta forma, la mujer estará en mejores condiciones para ver cuáles son sus alternativas reales. Eventualmente puede abrirse a otras opciones que no creía posibles o reevaluar una situación que le producía angustia bajo nuevos ojos.

Por último, el derecho a la autonomía de la paciente no debe entenderse instrumentalmente solo como una manera de asegurar un consentimiento informado respecto de la eventual interrupción o del tratamiento médico. Implica también tener las condiciones que permitan a la mujer vivir todo el proceso de una manera en que sus creencias, preferencias y valores sean respetados y tomados en consideración. Por ejemplo, es muy importante que sea la mujer la que decida si quiere ver o no la ecografía, si quiere tomar en brazos o no a su hijo mortinato, si desea o no hacer una ceremonia familiar para despedirlo o ponerle nombre. Las experiencias que viven las mujeres que se encuentran en estas tres causales y sus familias son, antes que nada, momentos de gran profundidad, intimidad e importancia existencial. Es deber del equipo de salud crear las condiciones para que las mujeres 
se sientan tratadas con dignidad y sus decisiones aceptadas e implementadas con delicado respeto.

\section{Conclusión}

El programa de acompańamiento contemplado en la Ley IVE exige que se respete el derecho a la autonomía de la mujer embarazada. Es voluntario, respetuoso de las decisiones de la paciente, personalizado, basado en información veraz y objetiva y no inductivo, ni a la decisión de mantener el embarazo ni a la de interrumpirlo. Los programas de acompañamiento que no cumplen con estas características vulneran el derecho a la autonomía de la paciente y son ilegales. El acompańamiento debe ofrecer condiciones de reconocimiento que permita a la mujer presentarse sin vergüenza, confiar en su propia competencia y hacerse responsable de sus decisiones. Debe proveer la información necesaria que permita a la mujer comprender sus opciones. El ejercicio de la autonomía requiere que el equipo de salud se involucre en un diálogo genuino con la paciente y se abstenga de imponer sus interpretaciones sobre la experiencia que la mujer está viviendo. Las creencias, preferencias y valores de la mujer deben guiar el proceso de toma de decisiones y deben ser respetadas en todas las etapas del acompañamiento.

Conflictos de interés: la autora no tiene conflictos de interés.

\section{Referencias}

1. Ley 21.030, que regula la despenalización voluntaria del embarazo en tres causales (septiembre 23, 2017).

2. Historia de la Ley 21.030. Disponible en: https://www.bcn.cl/historiadelaley/nc/historia-de-la-ley/6701/

3. Perrucci AC. Decision Assessment and Counseling in Abortion Care. Philosophy and Practice. New York: Rowman \& Littlefield; 2012.

4. Baker A, Beresford T. Informed Consent, Patient Education, and Counseling. In: Paul M, Lichtenberg S, Borgatta L, Grimes DA, Stubblefield PG, Creining MD, eds. Management of Unintended and Abnormal Pregnancy: Comprehensive Abortion Care. Oxford: Blackwell; 2009: 48-62.

5. Federal Ministry for Family Affairs, Senior Citizens, Women and Youth. Pregnancy Counselling $\$$ 218: Information on the Act on Assistance to Avoid and Cope with Conflicts in Pregnancy and Statutory Regulations Pertaining to Section 218 of the German Criminal Code; 2014. Disponible en: https://www.bmfsf.de/blob/95278/356f87878e2f128eb9aa85 c1451430fb/schwangerschaftsberatung---218-englisch-data.pdf

6. Tribunal Constitucional, Chile. Sentencia Rol No 3729 (3751)-17 CTP (agosto 28, 2017).

7. Waldron J. Second Lecture: Law, Dignity and Self-control. In: Waldron J. Dignity \& Rights. The Berkeley Tanner Lectures. Oxford: Oxford University Press; 2015: 51-53.

8. Undurraga V. Delineando una propuesta de reconocimiento del derecho a la autonomía personal en la nueva constitución. En: Mesa A, ed. Proceso Constituyente: Nueva Constitución. Actas XII Jornadas Constitucionales. Temuco: Universidad Mayor; 2016: 131-152.

9. Tribunal Constitucional, Chile. Sentencia Rol No 1683-10-INA. (enero 4, 2010).

10. Ley 20.584 que regula los derechos y deberes que tienen las personas en relación con acciones vinculadas a su atención en salud (abril 24, 2012).

11. Barcia R. La capacidad extrapatrimonial de los niños y adolescentes conforme a sus condiciones de madurez. Revista Ius et Praxis 2013; 19(2): 3-52.

12. Comité de Derechos del Niño. Observación General No4. La salud y el desarrollo de los adolescentes en el contexto de la Convención sobre los Derechos del Niño. CRC/GC/2003/4 (2003), párrafos 11, 31 y 33.

13. Decreto 44 del Ministerio de Salud. Aprueba Reglamento de las Prestaciones Incluidas en el Programa de Acompañamiento y Materias Afines de la Ley No 21.030 (febrero 8, de 2018).

14. Zúniga A. La nueva Ley de Derechos del Paciente: del modelo de la beneficencia al modelo de la autonomía. Revista Médica de Chile 2013; 141(1): 123-124.

15. Christman J, ed. The inner citadel: Essays on Individual Autonomy. Nueva York: Oxford University Press; 1989.

16. Christman J, Anderson J, eds. Autonomy and the challenges to liberalism: New Essays. Nueva York: Cambridge University Press; 2005.

17. Beauchamp TL, Childress JF. Principles of Biomedical Ethics. Seventh edition. New York: Oxford University Press; 2013. 
18. Kottow M. El consentimiento informado en clínica: inquietudes persistentes. Revista Médica de Chile Chile 2016; 144: 1459-1463.

19. Sherwin S. A Relational Approach to Autonomy in Health Care. In: Sherwin S, coord. The politics of Women's Health: Exploring Agency and Autonomy. Philadelphia: Temple University Press; 1998.

20. May T. The Concept of Autonomy in Bioethics: An Unwarranted Fall from Grace. In: Taylor JS, ed. Personal Autonomy. New Essays on Personal Autonomy and Its Role in Contemporary Moral Philosophy. Cambridge: Cambridge University Press; 2005: 299-309.

21. Smith JF. Communicative Ethics in Medicine: The Physician-Patient Relationship. In: Wolf SM, ed. Feminism \& Bieethics Beyond Reproduction. New York: Oxford University Press; 1996.

22. Cook RJ, Cusack S. Gender Stereotyping: Transnational Legal Perspectives. Philadelphia: University of Pennsylvania Press; 2010.

23. Federación Internacional de Ginecología y Obstetricia (FIGO). Recomendaciones sobre Temas de Ética en Obstetricia y Ginecología hechas por el Comité para el Estudio de los Aspectos Éticos de la Reproducción Humana de la FIGO; 2012. Disponible en: https://www.figo.org/sites/default/files/uploads/wg-publications/ethics/Spanish\%20Ethical\%20 Issues\%20in\%20Obstetrics\%20and\%20Gynecology.pdf

24. Olsen F. El sexo del derecho. En: Ruiz AEC. Identidad Femenina y Discurso Jurídico. Buenos Aires: Editorial Biblos, Colección Mujer y Derecho; 2000: 25-42.

25. Kessler RC, Schatzberg AF. Commentary on Abortion Studies of Steinberg and Finer (Social Science \& Medicine 20011; 72: 72-82) and Coleman (Journal of Psychiatric Research 2009; 43: 770-6 \& Journal of Psychiatric Research 2011; 45: 1133-4). Journal of Psychiatric Research 2012; 46(3), 410-411.

26. Nedelsky J. Law's relations: a relational theory of self, autonomy and law. New York: Oxford University Press; 2013.

27. Cook RJ. Significados Estigmatizados del Derecho Penal Sobre el Aborto. En: Cook RJ, Erdman JN, Dickens BM, eds. El aborto en el Derecho transnacional. Casos y Controversias. México: Fondo de Cultura Económica, Centro de Investigación y Docencia Económicas (CIDE); 2016, p. 438-467.

28. Cook RJ, Dickens BM. Reducing Stigma in Reproductive Health. International Journal of Gynecology and Obstetrics 2014: (125): 89-92.

29. Anderson J, Honneth A. Autonomy, Vulnerability, Recognition, and Justice. In: Christman J, Anderson J, eds. Autonomy and the Challenges to Liberalism: New Essays. New York: Cambridge University Press; 2005: 127-149.

Recibido: 12 de mayo de 2018

Aceptado: 15 de junio de 2018 